\title{
Uma sondagem via internet projetada para avaliar a vida diária dos idosos
}

\section{Resumo}

O declínio da saúde física e do funcionamento cognitivo, em decorrência da idade, pode ter efeitos profundos na vida diária dos idosos, inclusive no desempenho de tarefas de autocuidado e na participação em atividades sociais. No entanto, pouco ainda se sabe sobre essa relação, devido à falta de medidas adequadas para uma avaliação diária dessa população. Este artigo apresenta um projeto piloto interdisciplinar que desenvolveu medidas diárias de saúde, funcionamento físico, atividades de autocuidado e atividades sociais entre idosos. No estudo, 182 idosos (com idades entre 60 e 90 anos) participaram, através da internet, de uma breve ( 15 minutos) pesquisa de base, e de avaliações diárias, durante sete dias consecutivos. As medidas incluídas nesse estudo, e suas estatísticas descritivas, são apresentadas neste trabalho. Na conclusão são discutidas suas implicações para pesquisas futuras, incluindo os desafios envolvidos no uso da internet para a coleta de dados de idosos.

Palavras-chave: Sondagem via internet. Pesquisa social. Idosos. Saúde dos idosos. Medidas diárias de saúde

\footnotetext{
"Professora de Sociologia e Pesquisadora Associada Sênior do Center for Policy Research Syracuse University - (Estados Unidos). E-mail: jwilmoth@maxwell.syr.edu.

${ }^{* *}$ Professor de Desenvolvimento Humano e Estudos da Família e diretor do Center for Health Aging - Pensylvania State University (Estados Unidos). E-mail: mjs56@psu.edu.

${ }^{* * *}$ Pesquisadora do Center for Healthy Aging - Pennsylvania State University (Estados Unidos). E-mail: jam935@psu.edu.
} 


\section{A web-based survey designed to assess older adults' daily lives ${ }^{1}$}

\section{Abstract}

Age-related declines in physical health and cognitive functioning may have profound effects on the daily lives of older adults, including influencing the performance of self-care tasks and engagement in social activities. However, little is known about these relationships due to the lack of appropriate daily assessment measures in this population. This article describes an interdisciplinary pilot study that developed daily measures of health, physical functioning, self-care activities, and social activities among older adults. In the study, 182 older adults (ages 60-90) completed a brief ( $\sim 15$ minutes) online baseline survey and online assessments once each day for seven consecutive days. The measures included in this study and their descriptive statistics are presented. Then the implications for future research, including the challenges of using web-based technology to gather survey data from older adults, are discussed.

Keywords: Web-based survey. Social research. Ageing studies. Older adults. Health. Daily health assessment.

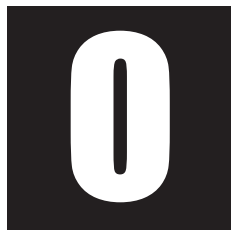

s gerontologistas, principalmente aqueles ligados à sociologia e à psicologia, têm examinado detalhadamente os diversos aspectos do bem-estar na terceira idade, incluindo o estado de saúde, o funcionamento físico e cognitivo, e a participação social. Muito já se sabe sobre esses tópicos, com dados representativos em âmbito nacional obtidos através de pesquisas em larga escala, anuais ou bianuais; bem como em estudos e experimentos realizados em pequena escala, com amostras não representativas. As medidas do estado de saúde e do funcionamen-

\footnotetext{
${ }^{1}$ Tradução de Marcelo Otto Severo.
} 
to físico nesses estudos normalmente incluem medidas padrões de autopercepção de saúde, capacidade funcional, e limitações nas atividades de vida diária (AVD) e nas atividades instrumentais de vida diária (AIVD) (Idler; Kasl, 1995; Lee, 2000; Katz et al., 1963; Nagi, 1965; Verbrugge; Jette, 1994). A maioria das pesquisas inclui medidas de funcionamento cognitivo baseadas em versões adaptadas do miniexame do estado mental (MEEM) (Folstein; Folstein; McHugh, 1975) ou da entrevista telefônica para avaliação do estado cognitivo (TICS: telephone interview of cognitive status) (Brandt; Spencer; Folstein, 1988). Da mesma forma, essas pesquisas costumam apresentar medidas gerais de satisfação com a vida, depressão, estresse psicológico, relações com a família e amigos, participação em atividades sociais e assistência por parte de cuidadores.

Embora essas medidas sejam bem desenvolvidas e validadas em diferentes populações, ainda se mostram problemáticas, por diversas razões. Primeiro, porque tendem a ser medidas relativamente globais de mudanças que ocorrem na terceira idade. O MEEM e o TICS, por exemplo, têm sido criticados porque só detectam uma disfunção cognitiva pouco expressiva e, portanto, são incapazes de caracterizar toda a gama de sutis alterações cognitivas que vêm com a idade (Lipton et al., 2003), ou a variação diária no funcionamento cognitivo que surge em resposta ao estresse do dia-a-dia (Sliwinski et al., 2006). Em segundo lugar, o longo período de referência dessas medidas - as últimas duas semanas ou mais - depende muito da lembrança precisa, o que pode ser prejudicado no caso de idosos e, sendo assim, podem relatar tendências que comprometem a sua validade (Carstensen; Hartel, 2006). Em terceiro lugar, essas medidas não observam a variabilidade intra-individual a curto prazo. Mesmo que possam acomodar mudanças por longos períodos - semanas, meses e anos - essas medidas assumem que os conceitos subjacentes são relativamente constantes por períodos de tempo mais curtos. No entanto, 
pode passar despercebida uma variação diária significativa capaz de afetar o bem-estar do idoso.

Os psicólogos interessados em compreender o funcionamento cognitivo, assim como o ânimo positivo e ânimo negativo, dependem de pesquisas diárias de cotidiano onde os entrevistados respondem a um breve levantamento todos os dias; e de projetos de medição nos quais os mesmos são observados longitudinalmente e avaliados várias vezes em cada ocasião de medição (Salthouse; Nesselroade, 2010; Sliwinski, 2008). Esses projetos têm apresentado resultados promissores. Mas, até agora, ainda não estão incluídas medidas diárias de saúde física ou participação social, justamente as que mais interessam aos sociólogos. Além disso, essas medidas não foram incorporadas, em larga escala, a conjuntos de dados nacionalmente representativos, que tendem a ser analisados por pesquisadores quantitativos na sociologia do envelhecimento.

O desenvolvimento de medidas diárias válidas de bem-estar e funcionamento do idoso, que possam ser usadas em pesquisas de populações, é um passo necessário para melhorar nossa compreensão da vida dos idosos em geral e da heterogeneidade que existe na terceira idade, principalmente no que diz respeito ao funcionamento físico e ao bemestar social. A perspectiva do curso de vida, amplamente citada na literatura da sociologia, reconhece que o envelhecimento é um processo que se desenrola ao longo do tempo. Diante disso, os estudiosos da sociologia do envelhecimento têm dedicado considerável atenção à identificação de circunstâncias do início da vida que influenciam a subsequente trajetória do meio para o fim, considerando questões de raça, classe e gênero (George, 2007). Mas a variação intra-individual na terceira idade, principal preocupação da psicologia do envelhecimento, tem sido largamente ignorada pelos sociólogos, que trabalham a partir de uma perspectiva do curso de vida. O que é lamentável, uma vez que o elemento intra-individual, 
ao longo de curtos períodos de tempo, pode influenciar a qualidade de vida diária e, potencialmente, prognosticar uma mudança a longo prazo na trajetória (Hultsch; MacDonald, 2004).

Consideremos, por exemplo, o funcionamento físico. Na ausência de uma crise no estado de saúde (um acidente grave, ataque cardíaco ou acidente vascular cerebral, etc.), é pouco provável que um idoso sofra uma queda repentina no funcionamento físico. Em vez disso, ele ou ela começa a ter "dias bons" e "dias ruins". Com o tempo, o equilíbrio entre "dias bons e ruins" muda, tornando-se cada vez mais difícil desempenhar papéis sociais e participar de atividades, o que poderia prejudicar o bemestar do idoso. Se uma pesquisa nacional longitudinal é conduzida uma vez por ano ou a cada dois anos, como costuma acontecer, as medidas só podem detectar mudanças no funcionamento a longo prazo. A flutuação intra-individual diária passa completamente despercebida - incluindo a medida em que esta varia entre os grupos, influencia a vida cotidiana e se relaciona com trajetórias de longo prazo. Além disso, qualquer tipo de avaliação individual (seja a cada dois anos, anualmente, ou mais frequente) corre o risco de avaliar um indivíduo em um dia "ruim", o que poderia comprometer as conclusões sobre o processo de envelhecimento. Distribuindo-se as avaliações por um período de dias sucessivos é possível desenvolver uma melhor estimativa do funcionamento real através da média entre dias bons e ruins.

Como ponto de partida, realizamos um projeto piloto concebido para desenvolver um conjunto de medidas diárias do cotidiano para a avaliação de atividades diárias, sintomas físicos, humor e experiências estressantes em idosos, o qual seria aplicado através da internet. Entre os objetivos específicos desse projeto estavam:

a) Validar o uso de procedimentos objetivos de avaliação cognitiva para idosos através da Internet; 
b) desenvolver e validar medidas de comportamentos diários que demonstram autocuidado e participação social; e

c) examinar as relações entre a pesquisa de base e os indicadores diários.

\section{O Projeto de Estudo}

A pesquisa de base. A primeira parte do estudo consistiu em uma pesquisa online para medidas gerais de saúde física, audição e visão, atividades físicas, atividades sociais e de lazer, e cognição. As questões foram dispostas em uma série de páginas sucessivas onde o entrevistado poderia apontar e clicar para gravar sua resposta a um determinado conjunto de questões, e então clicar em um botão "continuar" na parte inferior da página para avançar para o próximo conjunto de perguntas.

Na pesquisa de base, as primeiras perguntas foram sobre questões sociodemográficas, como idade, sexo, raça e etnia, escolaridade, renda atual, estado civil e número de filhos. A seguir, foram coletados dados sobre medidas de saúde, começando por uma medida global de autopercepção de saúde, onde 1 = ruim e 5 = excelente. Perguntamos então aos entrevistados "se algum médico já lhe disse que você teve alguma dessas" condições: ataque cardíaco, angina, insuficiência cardíaca congestiva ou outra doença cardíaca, artrite, osteoporose, fratura no quadril, diabetes, doença pulmonar, acidente vascular cerebral, demência/mal de Alzheimer, câncer, ou outra doença grave. Perguntamos também sobre os seguintes "problemas de saúde que você possa ter apresentado no mês passado": respiração, coração/circulação, estômago, costas/pescoço, limitação de força ou movimento, mastigar/engolir, falta de energia. Para cada condição indicada seguia-se uma questão sobre o quanto esse problema limitou a atividade durante o mês passado (de $1=$ todos os dias/sete dias 
por semana, até 5 = nunca). Os participantes deveriam então informar se usam aparelho auditivo ou óculos/lentes de contatos, e até que ponto eles podem realizar determinadas atividades com o aparelho auditivo ou óculos/ lentes. O último conjunto de perguntas sobre atividade física baseia-se nas escalas de resultados referidos pelos pacientes (PROMIS: Patient Reported Outcomes Measurement Information System), desenvolvidas pelo United States National Institutes of Health ${ }^{1}$. Essas escalas perguntam aos entrevistados o quanto os problemas de saúde limitam diferentes tipos de atividade física (incluindo atividades intensas, andar mais de um quilômetro, subir um lance de escadas, levantar ou carregar mantimentos, curvar-se/ajoelhar-se/ dobrar-se) e o quanto ele ou ela tem dificuldade para realizar atividades diversas (tarefas domésticas, vestir-se, lavar o cabelo, tomar banho e se secar, sentar-se e levantar-se do vaso sanitário).

A seguir, a pesquisa de base apresenta duas páginas que fazem uso das escalas sociais de PROMIS para avaliar a satisfação do indivíduo com atividades sociais e de autocuidado: o tempo gasto com atividades de lazer, seu nível atual de atividades com os amigos, seu nível atual de atividade social, o quanto eles podem trabalhar, sua capacidade para se divertir em casa, praticar atividades com os amigos, praticar atividades de lazer, divertir-se fora de casa, fazer as coisas para a família, atender às necessidades dos outros, cumprir sua rotina diária, realizar serviços, trabalhar, e executar tarefas domésticas.

Por último, o estudo contém três tarefas de cognição que foram coletadas na pesquisa de base. A primeira, conhecida como one back, é um teste de foco de atenção (Smith; Jonides, 1999), onde uma série de formas é apresentada na tela e o entrevistado clica no botão direito do mouse se ele/ela vê a forma duas vezes seguidas e no botão esquerdo se

\footnotetext{
${ }_{1}^{1}$ Para mais informações, ver http://www.nihpromis.org/default.
} 
duas formas consecutivas são diferentes. A segunda tarefa é um teste de decisão, correspondência numérica (Ekstrom; French; Harman; Dearman, 1976), no qual dois números são apresentados na tela e o indivíduo clica no botão direito do mouse se os números são os mesmos ou no botão esquerdo se os números são diferentes. O terceiro é um teste de memória de trabalho, span visuoespacial, concebido como uma versão computadorizada do teste de blocos de Corsi (Milner, 1971), onde a letra X aparece em um tabuleiro de $4 \times 4$ na tela e o entrevistado clica nas células onde ele/ela viu o $X$, na ordem em que foi visto.

A pesquisa de acompanhamento diário. A segunda parte do estudo consistiu em uma pesquisa de acompanhamento diário online, de aproximadamente 15 minutos por dia, durante sete dias. Optamos por acompanhar os entrevistados por sete dias porque estudos anteriores de acompanhamento diário do cotidiano, com o mesmo plano, mostraram que esse período de tempo oferece um número suficiente de observações para compreender a variação intra-individual nos processos de interesse, sem sobrecarregar o indivíduo (Stone; Kessler; Haythornthwaite, 1991). Embora fosse preferível acompanhar os entrevistados longitudinalmente ao longo de vários períodos de sete dias (um corte longitudinal, por exemplo; Nesselroade, 1991), tal abordagem estava além do âmbito do presente estudo piloto.

Assim como na pesquisa de base, as perguntas foram dispostas em uma série de páginas sucessivas, onde o entrevistado poderia apontar e clicar para gravar sua resposta a um determinado conjunto de questões e, então, clicar em um botão "continuar" na parte inferior da página para avançar para o próximo conjunto de perguntas. Os indivíduos poderiam logar-se para concluir a pesquisa diária entre as $16 \mathrm{~h}$ desse dia e as $2 \mathrm{~h}$ do dia seguinte (os horários foram ajustados para o fuso do participante). Essa bateria trazia questões relacionadas a estresse e humor, sintomas físicos, atividade física, atividades de autocuidado, e atividades sociais nas últimas 24 horas. 
O ânimo positivo e o ânimo negativo são medidos pelos 12 itens da Escala de Ânimo Positivo e Negativo (PANAS: Positive and Negative Affect Schedule; Watson; Clark, 1994), que pergunta: "O quanto você se sente ___ (feliz; tenso; calmo; triste; satisfeito; preocupado; estimulado; desapontado; entusiasmado; relaxado; envergonhado; entediado) hoje?" (de 1 = nem um pouco, até 7 = extremamente). Criamos uma escala de estresse percebido (Cohen; Williamson, 1988) com base em quatro itens: "Hoje, quantas vezes você sentiu que (fosse incapaz de controlar as coisas importantes em sua vida; estava confiante quanto a sua capacidade para lidar com seus problemas pessoais; as coisas não estavam acontecendo de acordo com a sua vontade; as dificuldades haviam se acumulado até o ponto em que você não poderia superá-las)?" (de 0 = nunca, até 4 = muito frequentemente). A escala de interferência cognitiva (Stawski; Mogle; Sliwinski, 2011) mede a capacidade de concentrar a atenção e o quanto um indivíduo é distraído por pensamentos intrusivos, com base em seis indicadores: "Hoje, quantas vezes você (tentou parar de pensar em problemas; pensou em algo que você não queria pensar; teve pensamentos que se mantinham recorrentes em sua cabeça; teve pensamentos que não conseguia impedir; fez coisas para se distrair dos seus pensamentos; manteve-se ocupado só para impedir os pensamentos de entrarem em seu mente)?" (de 1 = nenhuma, até 7 = muito frequentemente).

Também foi apresentada aos entrevistados uma lista de sintomas físicos (Larsen; Kasimatis, 1991) que poderiam ter experimentado nas últimas 24 horas, dentro de quatro categorias principais: dores e incômodos (dor nas costas, dor de cabeça, dor nas articulações, dor menstrual, dor muscular, dor de garganta); problemas estomacais (prisão de ventre ou diarreia, náuseas, falta de apetite, dificuldade para mastigar ou engolir); coração e pulmões (sintomas de alergia, dor ou aperto no peito, resfriado/ gripe, congestionamento, coração acelerado, dificuldade para respirar); e 
outros sintomas (tonturas, calor e frio, tremores ou abalos, outro sintoma). Para cada sintoma físico observado, os entrevistados foram questionados sobre o quanto esse sintoma interferiu ou restringiu suas atividades diárias durante o dia e a gravidade desses sintomas (ambos em uma escala onde 1 = nem um pouco, e 7 = extremamente).

Os participantes foram então questionados sobre o uso de equipamentos auxiliares de mobilidade nas últimas 24 horas: bengala, cadeira de rodas, andador, ou scooter. Em seguida, responderam a uma série de perguntas sobre atividade física. Primeiro, eles responderam se realizaram os seguintes movimentos físicos sem ajuda de outra pessoa ou qualquer equipamento: andar três ou mais quadras, subir dez ou mais degraus de escada, levantar ou carregar objetos de 4,5 kg², curvar-se, estender os braços sobre a cabeça, agarrar ou segurar pequenos objetos com os dedos. Para cada movimento físico relatado, o entrevistado foi questionado sobre o nível de dificuldade envolvido na realização do movimento (nenhum, um pouco, algum, muito). Depois, informaram se haviam praticado exercícios leves (ioga, pesca, caminhada, por exemplo); médios (caminhada rápida, baseball, tênis, ciclismo moderado, voleibol, natação leve, por exemplo); e vigorosos (corrida, jogging, futebol americano, futebol, natação intensa, por exemplo) nas últimas 24 horas. Todos os entrevistados foram questionados sobre o quanto a saúde física interferiu em sua capacidade de se exercitar e sobre seu nível de satisfação em relação a sua capacidade nos exercícios (de 1 = nem um pouco, até 5 = extremamente).

Os participantes foram orientados a indicar que atividades de autocuidado eles realizaram nas últimas 24 horas: autocuidado pessoal (tomar banho, lavar o cabelo, fazer a barba, escovar os dentes, pentear o cabelo, vestir-se, tomar medicamentos prescritos, tomar medicamentos de venda

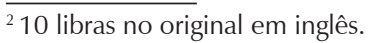


livre); preparação de refeições (café da manhã, almoço, jantar, lanches); limpeza e manutenção da casa (lavar louça, arrumar a cama, lavanderia, limpeza leve, limpeza pesada, jardinagem leve, jardinagem pesada, manutenção ou reparos leves, manutenção ou reparos pesados); e outras atividades (abrir a correspondência, preencher documentação, pagar contas, administrar o talão de cheques, gerenciar contas de poupança e investimento). Em seguida, eles responderam se praticaram as seguintes atividades de interesse social e pessoal nas últimas 24 horas: interações com familiares/amigos (visitar a família/vizinhos ou amigos, telefonar para a família/vizinhos ou amigos, entrar em contato com a família/vizinhos ou amigos através da Internet); frequentar eventos (cerimônia religiosa, evento esportivo, evento cultural, evento educacional, programa recreativo/reunião de clube, atividade em grupo, outro evento); trabalho e assistência (trabalho remunerado, trabalho voluntário, cuidar de criança menor de 18 anos, cuidar de adulto de 18 anos ou mais); atividades de lazer (ouvir rádio, assistir a TV, ir ao cinema, leitura, jogos de tabuleiro/ quebra-cabeças, jogar xadrez, fazer palavras cruzadas/sudoku/outros quebra-cabeças); e outras atividades (hobby/artesanato, golfe, boliche, dança, outros). Para cada conjunto de atividades, solicitou-se aos participantes que informassem o quanto a saúde física interfere em sua capacidade de realizar essas atividades (de $1=$ nem um pouco, até $5=$ extremamente), e sua satisfação em relação a sua capacidade de realizar essas atividades (de 1 = nem um pouco, até 5 = extremamente).

Recrutamento. $\mathrm{O}$ recrutamento de participantes para este projeto piloto foi um desafio. Era necessário recrutar indivíduos com acesso diário a um computador conectado à internet, uma vez que o estudo não dispunha de fundos suficientes para disponibilizar equipamentos para uma amostra aleatória de participantes. A estratégia padrão para alcançar a população com computadores é o recrutamento pela internet. Essa 
abordagem nem sempre produz amostras que sejam nacionalmente representativas, já que a possibilidade de o indivíduo ter um computador conectado à internet não está igualmente distribuída entre a população. Mas, por outro lado, pode produzir amostras representativas de usuários de internet, e fornecer um campo de teste para medidas que podem ser desenvolvidas em estudos futuros com o uso de metodologias alternativas que permitam o recrutamento de amostras representativas a nível nacional (levantamentos através de smartphones ou iPads, em que as consultas são enviadas na forma de "pacotes" com retorno pré-pago a indivíduos já cadastrados em um estudo de coorte).

Existem diversas empresas que fazem recrutamento online de amostras populacionais para participarem em pesquisas em ciências sociais. Inicialmente, pretendíamos recrutar participantes através do site Amazon's Mechanical Turk ${ }^{3}$, em que é possível publicar "tarefas" que devem ser preenchidas (uma pesquisa, por exemplo), às quais "trabalhadores" registrados podem inscrever-se e completá-las online, mediante remuneração. Logo percebemos que adultos de mais idade estavam sub-representados ( $<8 \%$ acima de 55 anos; Ross; Zaldivar; Irani; Tomlinson; Silberman, 2010) entre os "trabalhadores" do site, e que não seria possível obter a amostra planejada no prazo estabelecido para o projeto. Tentamos então recrutar participantes através do Survey Sampling Internationa ${ }^{4}$ (SSI), um serviço opt-in de amostragem que notifica pessoas interessadas sobre oportunidades de pesquisa, geralmente voltadas para o marketing. O SSI encaminha aos seus filiados um e-mail com os detalhes de um estudo em particular e estes decidem se desejam ou não participar do mesmo. Depois de uma primeira rodada de recrutamento, percebemos que o SSI

\footnotetext{
${ }^{3}$ https://www.mturk.com/mturk/welcome.

${ }^{4}$ http://www.surveysampling.com.
} 
foi projetado principalmente para pesquisas de mercado, e que não estava preparado para lidar com um estudo de medidas recorrentes. Diante disso, optou-se por recrutar os participantes através do SocialSci5 ${ }^{5}$ uma empresa de pesquisas na internet que auxilia pesquisadores em psicologia, sociologia e outras ciências sociais na realização de estudos online. O SocialSci tem parceria com uma série de residenciais geriátricos com o objetivo específico de prover pesquisadores com amostras de população idosa e, dessa forma, foi capaz de recrutar diretamente indivíduos na faixa etária apropriada para o presente estudo.

Ao cabo, conseguimos recrutar 182 participantes para o estudo. Entre esses indivíduos, a taxa de conclusão do acompanhamento diário foi a seguinte: $22 \%$ - 1 dia, $17 \%$ - 2 dias, $14 \%$ - 3 dias, a 12\% - 4 dias, $12 \%$ - 5 dias, 12\% - 6 dias, 11\% - 7 dias. Houve, portanto, uma abstenção significativa durante o período de sete dias do estudo, embora houvesse um incentivo financeiro para os indivíduos que completassem todos os oito questionários (isto é, a pesquisa de base mais os sete levantamentos diários). O protocolo aprovado pelo nosso Comitê de Ética permitiu que os participantes fossem compensados com base em seu nível de participação. Ao assinarem termo de consentimento informado, cada participante foi informado, por escrito, de que receberia $\$ 10$ por cada uma das pesquisas de que participasse, independentemente de o questionário ter sido totalmente concluído. Se o indivíduo completasse sete dos oito questionários dentro do prazo (8-10 dias), ele ou ela receberia um bônus adicional de $\$ 5$, enquanto que aqueles que completassem todos os oito questionários dentro do prazo (8-10 dias) receberiam um bônus adicional de $\$ 15$. Sendo assim, os indivíduos poderiam receber um total de $\$ 95$ se participassem de todas as oito sessões dentro do prazo. É importante no-

${ }^{5}$ https://www.socialsci.com. 
tar que as desistências variaram muito entre as iferentes fontes de recrutamento. Dos 117 participantes da amostra recrutados pelo SSI, somente $32 \%(n=37)$ forneceram dados para todas as oito avaliações, e 56\% completaram apenas uma ou duas sessões. Por outro lado, de um total de 65 indivíduos recrutados pelo SocialSci, 81\% $(n=56)$ completaram sete ou mais sessões. Isso mostra que o SocialSci é um mecanismo de recrutamento mais eficaz para esse tipo de pesquisa, dada a sua capacidade para lidar com estudos intensivos de medidas recorrentes.

\section{Estatística descritiva e bivariada}

As tabelas 1 e 2 apresentam as estatísticas descritivas da amostra para a pesquisa de base e as pesquisas diárias. No geral, a amostra de referência mostrou-se relativamente jovem (idade média $=67$ ), de maioria feminina (62\%), brancos nãohispânicos (93\%), e com boa escolaridade (47\% com faculdade ou pós-graduação). A maioria tinha pelo menos um filho (78\%). Curiosamente, boa parte dos entrevistados (64\%) não se mostrou disposta a revelar seus rendimentos. Vale destacar, também, que a autopercepção de saúde da amostra (média de 3,10 em uma escala onde 1 = fraco e 5 = excelente) está um pouco abaixo do que o normal em pesquisas nacionais com americanos idosos. A média para condições e problemas de saúde ficou ligeiramente acima de dois. São poucos os que precisam de aparelho auditivo (5\%), mas a maioria usa óculos para perto (81\%) e/ou para longe (73\%). O nível de limitações físicas e dificuldades, segundo a escala PROMIS, é relativamente baixo (média $=19,86$ ), enquanto que o grau de satisfação com as atividades de interesse social e pessoal, considerando-se a mesma escala, é relativamente alto (média $=53,99$ ). 
Tabela 1. Estatísticas descritivas da amostra da pesquisa de base

\begin{tabular}{|c|c|c|c|}
\hline Variável & Porcentagem & $\begin{array}{c}\text { Média } \\
\text { (desvio padrão) } \\
\end{array}$ & Faixa \\
\hline $\begin{array}{l}\text { Características sociodemográficas } \\
\text { Idade } \\
\text { Sexo (1 = feminino) } \\
\text { Raça/Etnia } \\
\text { Branca } \\
\text { Não-branca } \\
\text { Hispânica (de qualquer raça) } \\
\text { Escolaridade } \\
\text { Ensino Médio incompleto } \\
\text { Ensino Médio } \\
\text { Ensino Superior incompleto } \\
\text { Ensino Superior } \\
\text { Pós-graduação } \\
\text { Estado civil } \\
\text { Casado ou em união estável } \\
\text { Divorciado ou separado } \\
\text { Viúvo } \\
\text { Nunca foi casado } \\
\text { Um ou mais filhos } \\
\text { Renda atual } \\
\text { Menos de } \$ 20.000 \\
\$ 20.000 \text { - } 49.999 \\
\$ 50.000 \text { - } 99.999 \\
\$ 100.000 \text { ou mais } \\
\text { Não informado }\end{array}$ & $\begin{array}{c}62 \\
\\
93 \\
5 \\
3 \\
\\
3 \\
18 \\
31 \\
21 \\
26 \\
\\
53 \\
25 \\
15 \\
8 \\
78 \\
\\
16 \\
9 \\
8 \\
4 \\
64\end{array}$ & $66.97(5,54)$ & $60-90$ \\
\hline $\begin{array}{l}\text { Saúde Física } \\
\text { Autopercepção de saúde } \\
\text { Número de condições } \\
\text { Problemas de saúde } \\
\text { Usa aparelho auditivo } \\
\text { Usa óculos para perto } \\
\text { Usa óculos para longe }\end{array}$ & $\begin{array}{r}5 \\
81 \\
73 \\
\end{array}$ & $\begin{array}{l}3,10(1,01) \\
2,22(1,56) \\
2,19(1,97)\end{array}$ & $\begin{array}{l}1-5 \\
0-8 \\
0-7\end{array}$ \\
\hline $\begin{array}{l}\text { PROMIS } \\
\text { Limitações e dificuldade em atividades físicas } \\
\text { Satisfação social }\end{array}$ & & $\begin{array}{c}19,86(7,84) \\
53,99(14,60)\end{array}$ & $\begin{array}{l}10-49 \\
14-70\end{array}$ \\
\hline $\begin{array}{l}\text { Função cognitiva } \\
\text { One back } \\
\text { Número correto } \\
\text { Tempo médio de reação (milissegundos) } \\
\text { Correspondência numérica } \\
\text { Escore correto }<0,80 \\
\text { Tempo médio de reação (milissegundos) } \\
\text { Span visuoespacial } \\
\text { Número de Xs corretos }\end{array}$ & 17 & $\begin{array}{l}17,56(6,11) \\
1741(475)\end{array}$ & $\begin{array}{c}1-52 \\
594-4713\end{array}$ \\
\hline
\end{tabular}

${ }^{6}$ Entre os indivíduos com um escore correto maior do que ou igual a $80 \%$. 
Sociologias, Porto Alegre, ano 14, no 31, set./dez. 2012, p. 120-144

Tabela 2. Estatísticas descritivas da amostra das pesquisas de acompanhamento diário

\begin{tabular}{|c|c|c|c|}
\hline Variável & Porcentagem & $\begin{array}{c}\text { Média } \\
\text { (desvio padrão) }\end{array}$ & Faixa \\
\hline $\begin{array}{l}\text { Emoções e pensamentos } \\
\text { Ânimo positivo } \\
\text { Ânimo negativo } \\
\text { Estresse percebido } \\
\text { Interferência cognitiva } \\
\end{array}$ & & $\begin{array}{c}27,10(7,63) \\
11,28(6,15) \\
7,95(3,83) \\
12,12(7,89) \\
\end{array}$ & $\begin{array}{l}6-42 \\
6-42 \\
4-20 \\
6-42 \\
\end{array}$ \\
\hline $\begin{array}{l}\text { Sintomas físicos } \\
\text { Número total } \\
\text { Dores e incômodos } \\
\text { Problemas de estômago } \\
\text { Coração e pulmões } \\
\text { Outros sintomas } \\
\text { Auxiliares de mobilidade } \\
\text { Bengala } \\
\text { Cadeira de rodas } \\
\text { Andador } \\
\text { Scooter } \\
\text { Dificuldade em atividades físicas } \\
\text { Qualquer dificuldade } \\
\text { Andar três ou mais quadras } \\
\text { Subir dez ou mais lances de escadas } \\
\text { Levantar ou carregar um objeto de } 4,5 \mathrm{~kg}^{2} \\
\text { Curvar-se } \\
\text { Estender os braços sobre a cabeça } \\
\text { Usar os dedos para agarrar } \\
\text { Exercícios } \\
\text { Leves } \\
\text { Médios } \\
\text { Rigorosos }\end{array}$ & $\begin{array}{c}40 \\
4 \\
1 \\
0 \\
\\
6 \\
0 \\
1 \\
1 \\
\\
50 \\
14 \\
14 \\
13 \\
23 \\
11 \\
13 \\
46 \\
18 \\
5\end{array}$ & $0,53(0,72)$ & $0-3$ \\
\hline $\begin{array}{l}\text { Atividades de autocuidado (número) } \\
\text { Total } \\
\text { Pessoal } \\
\text { Preparação de refeições } \\
\text { Tarefas domésticas } \\
\text { Outra }\end{array}$ & & $\begin{array}{l}12,77(4,04) \\
5,21(1,36) \\
2,61(1,22) \\
2,87(1,78) \\
2,08(1,51)\end{array}$ & $\begin{array}{l}0-23 \\
0-7 \\
0-4 \\
0-6 \\
0-5\end{array}$ \\
\hline $\begin{array}{l}\text { Atividades de autocuidado (satisfação) } \\
\text { Pessoal } \\
\text { Preparação de refeições } \\
\text { Tarefas domésticas } \\
\text { Outra }\end{array}$ & & $\begin{array}{l}4,14(1,35) \\
4,15(1,40) \\
4,04(1,37) \\
4,32(1,30)\end{array}$ & $\begin{array}{l}1-5 \\
1-5 \\
1-5 \\
1-5\end{array}$ \\
\hline $\begin{array}{l}\text { Atividades sociais (número) } \\
\text { Total } \\
\text { Família e amigos } \\
\text { Eventos } \\
\text { Trabalho } \\
\text { Lazer } \\
\text { Outra }\end{array}$ & & $\begin{array}{l}6,84(3,14) \\
2,56(1,53) \\
0,39(0,68) \\
0,50(0,68) \\
2,86(1,04) \\
0,53(0,66)\end{array}$ & $\begin{array}{l}0-18 \\
0-6 \\
0-4 \\
0-4 \\
0-6 \\
0-2\end{array}$ \\
\hline $\begin{array}{l}\text { Atividades sociais (satisfação) } \\
\text { Família e amigos } \\
\text { Eventos } \\
\text { Trabalho } \\
\text { Lazer } \\
\text { Outra }\end{array}$ & & $\begin{array}{l}4,38(1,20) \\
3,93(1,55) \\
3,95(1,47) \\
4,30(1,25) \\
2,00(2,35)\end{array}$ & $\begin{array}{l}1-5 \\
1-5 \\
1-5 \\
1-5 \\
1-5\end{array}$ \\
\hline
\end{tabular}


Os entrevistados apresentam escores de funcionamento cognitivo típicos para uma população nessa faixa etária. Mas é importante ressaltar que $17 \%$ da amostra tinha uma porcentagem de correspondência numérica correta inferior a $80 \%$. De fato, muitos participantes $(n=6)$ obtiveram $30 \%$ ou menos de escores corretos. Dada a facilidade da tarefa, uma porcentagem muito baixa de correspondência numérica correta indica que os indivíduos podem estar marcando suas respostas de maneira contrária às instruções (seja aleatória ou sistematicamente). É possível que eles tenham confundido os botões do mouse durante a tarefa, ou que sejam canhotos e os botões estejam invertidos no computador. Em ambos os casos, a decisão do indivíduo está correta, mas ele ou ela está, sem querer, assinalando uma decisão incorreta ao clicar no botão errado do mouse. Considerando o fato de que essa tarefa é projetada para observar a precisão na tomada de decisões, queremos analisar somente os dados dos participantes dos quais podemos afirmar com alguma certeza que seguiram sistematicamente as instruções de uso do mouse. Por isso, recomendamos não analisar indivíduos com correspondência numérica correta menor do que $80 \%$, o que é uma base significativamente maior e mais conservadora do que os $50 \%$ que seriam de se esperar, mesmo considerando o acaso.

O acompanhamento diário indica que o ânimo positivo é maior do que o negativo nessa amostra (média $=27,10$ e 11,28, respectivamente), o que é consistente com outros levantamentos diários de ânimo nessa população (Carstensen; Pasupathi; Mayr; Nesselroade, 2000). O estresse percebido e a interferência cognitiva também são relativamente baixos (média $=7,95$ e 12,12, respectivamente), semelhante ao que foi observado em levantamentos diários anteriores (Stawski; Mogle; Sliwinski, 2011). Os participantes relatam poucos sintomas físicos diários. A média é inferior a um, e a extensão total é de zero a três. No entanto, $40 \%$ dos entrevistados relatam sentir dores e incômodos. São poucos os que se utilizam de equipamentos auxiliares de mobilidade, como bengala (6\%), 
andador (1\%), ou scooter (1\%). A maioria (50\%) apresenta algum grau de dificuldade em pelo menos uma das seis atividades físicas, sendo que a mais comum é a flexão (23\%). Curiosamente, quase a metade (48\%) e um quinto (18\%) afirma praticar exercícios leves e médios, respectivamente. Apenas 5\% praticam atividades físicas intensas. Os participantes apresentam um alto nível de participação em atividades de autocuidado, com uma média diária de 12,77, e um nível elevado de satisfação com a sua capacidade de se envolver nessas atividades. Os indivíduos também estão bastante envolvidos em atividades de interesse social e pessoal, com uma média diária de 6,84; mas eles parecem estar menos satisfeitos com sua capacidade de participar de atividades relacionadas a eventos, trabalho e outros interesses do que atividades que envolvam lazer e família/amigos.

Como pode ser visto na Tabela 3, há uma forte correlação entre conceitos similares na pesquisa de base e nas medidas diárias de saúde, funcionamento físico e atividades sociais. A referência de autopercepção de saúde está negativamente relacionada a sintomas físicos diários $(r=$ $-0,26)$ e à dificuldade em atividades físicas $(r=-0,31)$. A referência de condições e problemas de saúde está positivamente associada a sintomas físicos diários ( $r=0,06$ e 0,33, respectivamente) e à dificuldade em atividades físicas diárias ( $r=0,14$ e 0,40, respectivamente). É interessante notar que os problemas de saúde estão mais fortemente associados a sintomas físicos e dificuldades em atividades físicas diárias do que às condições. A referência da escala PROMIS para limitações e dificuldade em atividade física está positivamente associada a sintomas físicos diários ( $r=$ $0,28)$ e à dificuldade em atividade física diária $(r=0,31)$; enquanto que a referência da mesma escala para satisfação social está positivamente associada à satisfação diária com atividades de autocuidado $(r=0,44)$ e à satisfação diária com atividades sociais $(r=0,49)$. 
Sociologias, Porto Alegre, ano 14, no 31, set./dez. 2012, p. 120-144

Tabela 3. Correlações entre dados selecionados e medidas diárias

\begin{tabular}{|c|c|c|c|c|c|c|}
\hline & \multicolumn{6}{|c|}{ Medidas de base } \\
\hline & $\begin{array}{l}\text { Sintomas } \\
\text { físicos } \\
\text { (número) }\end{array}$ & $\begin{array}{c}\text { Qualquer } \\
\text { dificuldade } \\
\text { em atividades } \\
\text { físicas }\end{array}$ & $\begin{array}{l}\text { Ativida- } \\
\text { des de } \\
\text { autocuidado } \\
\text { (número) }\end{array}$ & $\begin{array}{c}\text { Ativida- } \\
\text { des de } \\
\text { autocuidado } \\
\text { (satisfação) }\end{array}$ & $\begin{array}{l}\text { Atividades } \\
\text { sociais } \\
\text { (múmero) }\end{array}$ & $\begin{array}{l}\text { Atividades } \\
\text { sociais } \\
\text { (satisfação) }\end{array}$ \\
\hline $\begin{array}{l}\text { Medidas de base } \\
\text { Saúde física } \\
\text { Autopercepção de } \\
\text { saúde } \\
\text { Número de con- } \\
\text { dições } \\
\text { Problemas de saúde }\end{array}$ & $\begin{array}{c}-0,26 \\
0,06^{\text {ns }} \\
0,33\end{array}$ & $\begin{array}{c}-0,31 \\
0,14 \\
0,40\end{array}$ & $\begin{array}{c}0,27 \\
-0,12 \\
-0,26\end{array}$ & $\begin{array}{c}0,31 \\
-0,22 \\
-0,17\end{array}$ & $\begin{array}{r}0,35 \\
-0,15 \\
-0,16\end{array}$ & $\begin{array}{c}0,44 \\
-0,26 \\
-0,27\end{array}$ \\
\hline $\begin{array}{l}\text { Funcionamento } \\
\text { cognitivo } \\
\text { One back } \\
\text { Número correto } \\
\text { Tempo médio de } \\
\text { reação } \\
\text { (milisegundos) }_{\text {Correspondência }} \\
\text { numérica } \\
\text { Tempo médio de } \\
\text { reação } \\
\text { (milisegundos)a }^{\text {Span visuoespacial }} \\
\text { Número de Xs } \\
\text { corretos }\end{array}$ & $\begin{array}{c}-0,03^{\mathrm{ns}} \\
-0,01^{\mathrm{ns}} \\
0,11 \\
0,002^{\mathrm{ns}}\end{array}$ & $\begin{array}{c}-0,11 \\
0,007^{\text {ns }} \\
0,14 \\
-0,07\end{array}$ & $\begin{array}{l}-0,06^{\mathrm{ns}} \\
-0,01^{\mathrm{ns}} \\
-0,18 \\
0,10\end{array}$ & $\begin{array}{c}0,09 \\
0,05^{\mathrm{ns}} \\
-0,22 \\
0,10\end{array}$ & $\begin{array}{l}-0,02^{\mathrm{ns}} \\
-0,01^{\mathrm{ns}} \\
-0,03^{\mathrm{ns}} \\
0,09\end{array}$ & $\begin{array}{l}0,05^{\mathrm{ns}} \\
0,07^{\mathrm{ns}} \\
-0,26 \\
-0,02^{\mathrm{ns}}\end{array}$ \\
\hline $\begin{array}{l}\text { Sintomas físicos } \\
\text { (número) }\end{array}$ & & 0,25 & $-0,04^{\mathrm{ns}}$ & $-0,23$ & $-0,03^{\mathrm{ns}}$ & $-0,27$ \\
\hline $\begin{array}{l}\text { Dificuldade em } \\
\text { atividades físicas }\end{array}$ & 0,25 & & $-0,08$ & $-0,13$ & $-0,13$ & $-0,10^{\mathrm{ns}}$ \\
\hline $\begin{array}{l}\text { Autocuidado } \\
\text { (número) }\end{array}$ & $-0,04^{\mathrm{ns}}$ & $-0,08$ & & 0,29 & $-0,52$ & 0,47 \\
\hline $\begin{array}{l}\text { Autocuidado (satis- } \\
\text { fação) }\end{array}$ & $-0,23$ & -13 & 0,29 & & 0,23 & 0,87 \\
\hline $\begin{array}{l}\text { Atividades sociais } \\
\text { (número) }\end{array}$ & $-0,03^{\mathrm{ns}}$ & $-0,13$ & 0,52 & 0,23 & & 0,49 \\
\hline $\begin{array}{l}\text { Atividades sociais } \\
\text { (satisfação) }\end{array}$ & $-0,27$ & $-0,10^{\mathrm{ns}}$ & 0,47 & 0,87 & 0,49 & \\
\hline
\end{tabular}

ns Estatisticamente não-significativo, $\mathrm{p}>0,05$.

${ }^{a}$ Entre os indivíduos com um escore correto maior do que ou igual a $80 \%$. 
Os resultados na Tabela 3 mostram também que as medidas de saúde, funcionamento físico, funcionamento cognitivo e emoções/pensamentos estão relacionadas à participação diária em atividades de autocuidado e sociais, e à satisfação com as mesmas. Geralmente, uma melhor referência de autopercepção de saúde está positivamente associada ao envolvimento diário $(r=0,27$ e $r=0,25)$ e à satisfação com $(r=0,31$ e 0,44 ) atividades de autocuidado e sociais, respectivamente; enquanto que mais referências de condições e problemas de saúde estão negativamente relacionadas ao envolvimento diário em atividades de autocuidado $(r=-0,13$ e $r=-0,26)$ e sociais $(r=-0,15$ e $r=-0,16)$, bem como à satisfação diária com atividades de autocuidado $(r=-0,22$ e $r=-0,17)$ e sociais ( $r=-0,26$ e $r=-0,27)$. A escala PROMIS de satisfação está positivamente associada ao número diário de atividades de autocuidado $(r=0,42)$ e atividades sociais $(r=0,31)$. As medidas de referência do funcionamento cognitivo não se mostraram tão fortemente relacionadas à participação diária em atividades de autocuidado e sociais, e à satisfação com as mesmas. Uma exceção é o tempo de reação da correspondência numérica, que está negativamente associado ao número diário de atividades de autocuidado $(r=-0,18)$, à satisfação com essas atividades $(r=$ $-0,22)$, e à satisfação com atividades sociais $(r=-0,26)$.

Esses resultados indicam que os participantes com reações mais rápidas (um identificador de melhor funcionamento cognitivo) estavam mais propensos a participar e a obter uma maior satisfação com a sua capacidade de participar dessas atividades diárias. O ânimo positivo diário está positivamente associado ao envolvimento e à satisfação com atividades diárias de autocuidado ( $r=0,20$ e $r=0,26$ ) e sociais ( $r=0,29$ e 0,37 ); enquanto que as correlações de ânimo negativo diário, estresse percebido e interferência cognitiva se mostram negativas (variando de elevados -0,30 na relação entre estresse percebido e satisfação com atividades sociais, até relações não- significativas entre a interferência cognitiva e o número de atividades 
de autocuidado e sociais). Os sintomas físicos diários e a dificuldade em atividades físicas também estão negativamente associados à participação diária e à satisfação com atividades de autocuidado e sociais (variando de elevados - 0,27 na relação entre sintomas físicos e satisfação com atividades sociais, até relações não-significativas entre os sintomas físicos e o número de atividades de autocuidado, bem como o de atividades sociais).

Finalmente, é interessante mencionar que os sintomas físicos diários e a dificuldade em atividades físicas estão negativamente associados ao ânimo positivo ( $r=-0,22$ e $-0,13$, respectivamente); e positivamente associados ao ânimo negativo ( $r=0,24$ e 0,06, respectivamente), ao estresse percebido $(r=0,25$ e 0,10$)$, e à interferência cognitiva $(r=0,27$ e 0,09 , respectivamente). Além disso, o número informado de atividades está positivamente associado à satisfação com essas atividades $(r=0,29$ para atividades de autocuidado e satisfação; $r=0,49$ para atividades sociais e satisfação). E, para concluir, o número de atividades de autocuidado está positivamente associado ao número de atividades sociais $(r=$ 0,52); e a satisfação com as atividades de autocuidado está positivamente associada à satisfação com as atividades sociais $(r=0,87)$.

\section{Discussão}

Este projeto piloto nos permitiu investigar a viabilidade da inclusão de medidas coletadas na internet em pesquisas de larga escala, geograficamente dispersas, com amostras obtidas através de amostragem aleatória. Não surpreende o fato de o recrutamento pela internet ter produzido uma amostra de idosos relativamente jovens, saudáveis, e com escolaridade alta. Consequentemente, esses resultados não podem ser generalizados para a população de idosos dos Estados Unidos. Ainda assim, a primeira fase deste projeto propicia uma validação inicial de nossa abordagem para o estudo 
do cotidiano de idosos. Nossa análise indica que a pesquisa de base e os levantamentos diários estão intimamente relacionados. Além disso, há uma forte correlação entre as variáveis do estudo e a participação e satisfação diária com atividades de autocuidado e sociais. Embora as tarefas de funcionamento cognitivo não estivessem tão fortemente associadas às medidas diárias como havíamos antecipado, uma análise suplementar (não mostrada) indica que a cognição está associada às emoções e pensamentos diários, o que está de acordo com os resultados de pesquisas anteriores (Sliwinski et al., 2006; Stawski; Mogle; Sliwinski, 2011). Dando continuidade à análise dos dados, vamos explorar mais detalhadamente a relação entre a pesquisa de base e as medidas diárias de saúde física, deficiência, e atividades. Precisamos também considerar mais cuidadosamente a variabilidade intraindividual nas medidas diárias.

Agora que foram desenvolvidas medidas diárias de cognição e comportamentos, pretendemos partir para uma segunda fase, com o objetivo de desenvolver uma validação adicional das medidas e métodos alternativos para executar a pesquisa por computador. Esforços anteriores para incorporar uma pesquisa pela internet no Estudo de Saúde e Aposentadoria (Health and Retirement Study) (Couper et al., 2007) mostraram que as taxas de uso da internet por idosos são baixas, e que aqueles que têm acesso à rede são diferentes dos que não têm, em uma gama de variáveis demográficas, financeiras, e de saúde. Isso sugere que as amostras de idosos usuários de internet não devem ser consideradas representativas da população dessa faixa etária, o que era o caso neste projeto piloto. Portanto, na segunda fase deste projeto vamos estudar a implantação de métodos de acesso à internet, como o empréstimo de computadores de baixo custo aos participantes ou a adaptação de dispositivos de mão (como o Nintendo DS ou os smartphones) para o uso dos mesmos. 
A expectativa de resultados a longo prazo deste projeto piloto é significativa. A adaptação aos desafios do envelhecimento ocorre dentro dos indivíduos, em tempo real. Uma análise das respostas ao estresse diário proporcionaria uma maior compreensão do fluxo e refluxo das limitações de saúde, frustrações, e solução de problemas do dia-a-dia, o que poderia se perder em uma pesquisa restrita ao estudo dos principais eventos e transições da vida (Almeida, 2005). Além de oferecer um insight sobre a vida quotidiana dos idosos, a inclusão de medidas como essas em pesquisas de populações permitirá à gerontologia mapear a vida diária em diferentes transições e trajetórias do desenvolvimento. Por exemplo, adicionar um levantamento diário do cotidiano a uma pesquisa como a do Estudo de Saúde e Aposentadoria, ou a do recente National Survey of Disability Trends and Dynamics, tornaria possível entender falhas cognitivas diárias e dificuldades relacionadas à saúde em grupos raciais/étnicos. Permitiria também aos pesquisadores identificar como essas falhas cognitivas e restrições de saúde interferem com a capacidade de participar de atividades diárias de autocuidado e sociais, para pesquisarem outros meios de auxiliar nas atividades de vida diária, mudanças nas condições de vida, e declínio na saúde a longo prazo.

\section{Referências}

1. ALMEIDA, D.M. Resilience and vulnerability to daily stressors assessed via diary methods. Current Directions in Psychological Science, v. 14, n. 2, p. 64-68, 2005.

2. BRANDT, J; SPENCER, M; FOLSTEIN, M. The Telephone Interview for Cognitive Status. Neuropsychiatry, Neuropsychology, and Behavioral Neurology, v. 1, n. 2, p. 111-117, 1988.

3. CARSTENSEN, L. L.; HARTEL, C.R. (Eds.) When I'm 64. Washington, DC: The National Academies Press, 2006. Committee on Aging Frontiers in Social Psychology, Personality, and Adult Developmental Psychology; Board on Behavioral, Cognitive, and Sensory Sciences; Division of Behavioral and Social Sciences and Education. 
4. CARSTENSEN, L. L.; PASUPATHI, M.; MAYR, U.; NESSELROADE, J. R. Emotional experience in everyday life across the adult life span. Journal of Personality and Social Psychology, v. 79, n. 4, p. 644-655, 2000.

5. COHEN, S.; WILLIAMSON, G. Perceived Stress in a Probability Sample of the United States. In: SPACAPAN, S.; OSKAMP, S. (Eds.) The Social Psychology of Health. Newbury Park, CA: Sage, 1988.

6. COUPER, M.P. et. alNoncoverage and nonresponse in an internet survey. Social Science Research, v. 36, n. 1 p. 131-148, Mar. 2007.

7. EKSTROM, R. B.; FRENCH, J. W.; HARMAN, H.; DERMAN, D. Manual for Kit of factor-referenced cognitive tests. rev. ed. Princeton, NJ: Educational Testing Service, 1976.

8. FOLSTEIN, M. F., FOLSTEIN, S. E., MCHUGH, P. R. "Mini-mental state". A practical method for grading the cognitive state of patients for the clinician. Journal of psychiatric research, v. 12, n. 3, p. 189-98, Jan. 1975.

9. GEORGE, L. Age structures, aging, and the life course. In: WILMOTH, J. M; FERRARO, K. F. Gerontology: Perspectives and Issues, $4^{\text {th }}$ Ed. New York: Springer, 2007. Chapter 8.

10. HULTSCH, D. F.; MACDONALD, S. W. S. Intraindividual variability in performance as a theoretical window onto cognitive aging. In: DIXON, R. A.; BÄCKMAN, L.; NILSSON, L-G. (Eds.) New frontiers in cognitive aging. Oxford: Oxford University Press, 2004. p. 65-88.

11. IDLER, E. L.; KASL, S. L. Self-ratings of health: Do they also predict change in functional ability? Journals of Gerontology: Social Sciences, v. 50B, n. 6, p. S344-S353, 1995.

12. KATZ, S. et al. Studies of illness in the aged. The index of ADL: A standardized measure of biological and psychosocial function. Journal of the American Medical Association, v. 185, p. 914-919, Sept. 1963.

13. LARSEN, R. J.; KASIMATIS, M. Day-to-day physical symptoms: Individual differences in the occurrence, duration, and emotional concomitants of minor daily illnesses. Journal of Personality, v. 59, n. 3, p. 387-423, Sept. 1991.

14. LEE, Y. The predictive value of self-assessed general, physical, and mental health on functional decline and mortality in older adults. Journal of Epidemiology and Community Health, v. 54, n. 2, p. 123-129, 2000.

15. LIPTON, R. et al. Screening for Dementia by Telephone Using the memory impairment screen. Journal of the American Geriatrics Society, v. 51, n. 10, p. 1382-1390, Oct. 2003. 
16. MILNER, B. Interhemispheric differences in the localization of psychological processes in man. British Medical Bulletin, v. 27, n. 3, p. 272-277, 1971.

17. NAGI, S. Some conceptual issues in disability and rehabilitation. In: SUSSMAN, M. (Ed.) Sociology and Rehabilitation. Washington, DC: American Sociological Association, 1965. p. 100-113.

18. NESSELROADE, J. R. The warp and woof of the developmental fabric. In: Downs, R.; Liben, L.; Palarmo, D. (Eds.), Visions of development, the environment, and aesthetics: The legacy of Joachim F. Wohlwill. Hillsdale, NJ: Erlbaum, 1991. p. 213-240.

19. ROSS, J.; ZALDIVAR, A.; IRANI, L.; TOMLINSON, B.; SILBERMAN, M.S. Who are the crowdworkers? Shifting demographics in Mechanical Turk. In: CHI CONFERENCE ON HUMAN FACTORS IN COMPUTER SYSTEMS, 28., Proceedings... Atlanta, GA: ACM, Apr. 2010. p. 2863- 2872.

20. SALTHOUSE, T. A.; NESSELROADE, J. R. Dealing with Short-term Fluctuation in Longitudinal Research. Journals of Gerontology: Psychological and Social Sciences, v. 65B, n. 6, p. 698-705, Nov. 2010.

21. SLIWINSKI, M. J. Measurement-burst designs for social health research. Social and Personality Psychology Compass, v. 2, n. 1, p. 245-261, Jan. 2008.

22. SLIWINSKI, M. J. et al. Intraindividual coupling of daily stress and cognition. Psychology and Aging, v. 21, n. 3, p. 545-557, 2006.

23. SMITH, E.; JONIDES, J. Storage and executive processes in the frontal lobes. Science's Compass, v. 283, n. 5408, p. 1657-1661, Mar. 1999.

24. STAWSKI, R.; MOGLE, J.; SLIWINSKI, M. Intraindividual coupling of daily stressors and cognitive interference in old age. The Journals of Gerontology: Psychological Sciences and Social Sciences, v. 66B, n. 1, p. i121-i129, July 2011.

25. STONE, A. A.; KESSLER, R. C.; HAYTHORNTHWAITE, J. A. Measuring daily events and experiences: Decisions for the researcher. Journal of Personality, $v$. 59, n. 3, p. 575-607, Sept. 1991.

26. WATSON, D.; CLARK, L. Manual for the Positive and Negative Affect Schedule. lowa: University of lowa Press, 1994.

Recebido em: 18/05/2012

Aceite final: 01/08/2012 